\title{
The utility of electrospun nanofibre mats for in vitro germination of Artabotrys hexapetalus pollen
}

\author{
Kitti Bodhipadma ${ }^{\mathrm{a}}$, Sompoch Noichinda ${ }^{\mathrm{a}}$, Noppavan Chanunpanich ${ }^{\mathrm{b}}$, Wisuda Sukthavornthum ${ }^{\mathrm{a}}$, \\ David W.M. Leung, ${ }^{\mathrm{c}, *}$ \\ ${ }^{a}$ Division of Agro-Industrial Technology, Faculty of Applied Science, \\ King Mongkut's University of Technology North Bangkok, Bangsue, Bangkok 10800 Thailand \\ b Department of Industrial Chemistry, Faculty of Applied Science, \\ King Mongkut's University of Technology North Bangkok, Bangsue, Bangkok 10800 Thailand \\ c School of Biological Sciences, University of Canterbury, Private Bag 4800, Christchurch 8140, \\ New Zealand
}

*Corresponding author, e-mail: david.leung@canterbury.ac.nz

Received 19 Mar 2015

Accepted 16 May 2016

\begin{abstract}
This study investigates the influence of electrospun fibre mats on in vitro pollen germination of Artabotrys hexapetalus (L.f.) Bhandari (synonym: A. odoratissimus R. Brown). There was no difference in the size of the pollen from two closely related stages of flower development, namely the yellow florets and yellowish orange florets. On agarbased medium supplemented with $20 \%$ sucrose, about $50 \%$ of the pollen from the yellowish orange florets germinated but only $25 \%$ of those from the yellow florets did. More pollen from the yellowish orange florets germinated in liquid medium than on agar-gelled medium but pollen tube elongation was greater in agar-gelled medium. Electrospinning of synthetic polymer solutions of polyvinylidene fluoride (PVDF) or polylactic acid (PLA) was used to generate mats composed of nonwoven (randomly oriented) nanofibres as well as those composed of a mixture of nonwoven and aligned (PVDF4 and PLA4) nanofibres. Compared with liquid, agar medium, and other types of nanofibre mats, PVDF4 was found to be the best physical support substrate for germination of the pollen from the yellowish orange florets (70\%) and tube growth (about 400\% increase over that in liquid medium). Pollen germination was largely inhibited on all four types of $18 \mu \mathrm{m}$ thick nanofibre mats but not on the $8 \mu \mathrm{m}$ thick ones.
\end{abstract}

KEYWORDS: pollen viability, pollen tube growth, polylactic acid (PLA), polyvinylidene fluoride (PVDF)

\section{INTRODUCTION}

In vitro pollen germination tests are useful for assessing pollen viability and performance independent of the influence of the female reproductive system ${ }^{1}$. The use of liquid or agar-gelled medium with the various ingredients is the most common physical support to germinate pollen in vitro. Earlier investigations have shown the importance of different ingredients including $\mathrm{Ca}^{2+}$ ions $^{2}$, boron ${ }^{3}$, and sucrose ${ }^{4}$ added to media for pollen germination of different plant species in vitro. A recent study shows that a solid support matrix such as a cellulose membrane placed on top of agarose gel resulted in a high percentage of Arabidopsis pollen germination in vitro ${ }^{5}$. Nevertheless, for each plant species it might be necessary to investigate the most appropriate method to get a high percentage of germination and good pollen tube growth.

Artabotrys hexapetalus (L.f.) Bhandari (Syn- onym: A. odoratissimus R. Brown), an indigenous plant of Sri Lanka and the southern part of India, was introduced from China to Thailand for ornamental use. It bears profusely year-round extremely fragrant flowers which are sought after for perfume production, and is easy to propagate and maintain. Floral ontogeny, anther, and seed development of A. hexapetalus have been studied extensively ${ }^{6-9}$, but the requirements for pollen germination in vitro of this Annonaceous plant have not been determined. This information might be helpful for assessing its reproductive system. In particular, the germination requirement of $A$. hexapetalus pollen was studied as a part of a larger study to build a better understanding as to why $A$. hexapetalus does not set fruit easily compared to other closely related species.

Electrospinning is a relatively simple technique to convert droplets of a polymer solution, under the influence of a high-voltage electrical field, into fibres of nanometres in diameter that can be collected in 
Table 1 Electrospinning conditions for generation of PVDF and PLA nanofibre mats.

\begin{tabular}{lccccc}
\hline Polymer & $\begin{array}{c}\text { Concentration } \\
(\%, \mathrm{w} / \mathrm{w})\end{array}$ & Solvent & $\begin{array}{c}\text { Voltage power supply } \\
(\mathrm{kV})\end{array}$ & $\begin{array}{c}\text { Travelling distance } \\
(\mathrm{cm})\end{array}$ & $\begin{array}{c}\text { Flow rate } \\
(\mathrm{ml} / \mathrm{h})\end{array}$ \\
\hline PVDF & 19 & DMAc/acetone 4:6 & 10 & 10 & 1 \\
PLA & 12 & THF/DMF 75:25 & 8 & 10 & 1 \\
\hline
\end{tabular}

the form of a fibrous mat. Conventional nanofibre mats consist of fibres deposited in a random (nonwoven) pattern or in alignment with a particular predetermined pattern. It was shown that fibre alignment in the electrospun fibrous mats plays an important role on the microenvironment permissive for human nerve cell growth ${ }^{10}$. Recently, electrospun nanofibre mats consisting of a combination of both nonwoven fibres and those aligned to a specific pattern ${ }^{11}$ were used as a novel physical support in a tissue culture medium for induction and growth of bilimbi callus ${ }^{12}$. It was found that different types of electrospun nanofibre mats had different effects on the growth of bilimbi callus. There is also paucity of information on the possible influence of different physical supports for pollen germination in A. hexapetalus. The present work is aimed to investigate if electrospun nanofibre mats would be useful physical support matrices for germination of A. hexapetalus pollen.

\section{MATERIALS AND METHODS}

\section{Pollen source}

Pollen grains were collected from the dehiscent anthers of yellow and yellowish orange flowers of the A. hexapetalus plants grown in the campus of King Mongkut's University of Technology North Bangkok, Thailand. The pollen grains from flowers of the two developmental stages were collected separately and care was taken to ensure that there was no mixing of the pollen from the two stages of flower development during the experiment. A digital camera (Olympus C-760, Olympus Corporation) was mounted on a light microscope (ML2000, Meiji Techno Co., Ltd., Japan) for taking all the images of pollen germination and pollen tube lengths in this study.

\section{Generation of nanofibre mats}

Chemicals: polyvinylidene fluoride, Kynar 761 (PVDF) and polylactic acid, NatureWorks grade 4042D (PLA) were obtained from Benebiz Co., Ltd. (Bangkok) and NatureWorks LLC (Minnetonka, MN, USA), respectively. $N, N^{\prime}$-dimethylacetamide (DMAc) and acetone were obtained from Fluka.
Tetrahydrofuran (THF) and $N, N^{\prime}$-dimethylformamide (DMF) were obtained from Sigma Aldrich.

Electrospinning: PVDF and PLA nanofibre mats were electrospun using the concentrations of the respective polymer solutions under the processing conditions (Table 1).

Nonwoven nanofibre mats were electrospun on an aluminium (Al) sheet mounted on a rotating drum, and were referred to herewith as PVDF1 or PLA1 (Fig. 1). In addition, nanofibre mats composed of a mixture of nonwoven and aligned fibres were also produced under the same condition but first with an $\mathrm{Al}$ sheet, and then with a designed patterned plastic (Petty patent number 6223 issued by Department of Intellectual Property, Ministry of Commerce, Nonthaburi, Thailand) were used. The nanofibre mats produced in this way were referred herewith as PVDF4 or PLA4. At the completion of electrospinning, the nanofibre mats were released from the $\mathrm{Al}$ sheets and designed patterned plastics. Then the disks of nanofibre mats of either $8 \pm 2 \mu \mathrm{m}$ or $18 \pm 2 \mu \mathrm{m}$ thickness were punched using a paper hole puncher of $25 \mathrm{~mm}$ in diameter before they were laminated with plastic o-rings. The working surface area of the nanofibre mats was $21 \mathrm{~mm}$ in diameter. Samples of the nonwoven PVDF and PLA nanofibre mats were sent to the Scientific and Technological Research Equipment Centre, Chulalongkorn University for scanning electron microscope analysis.

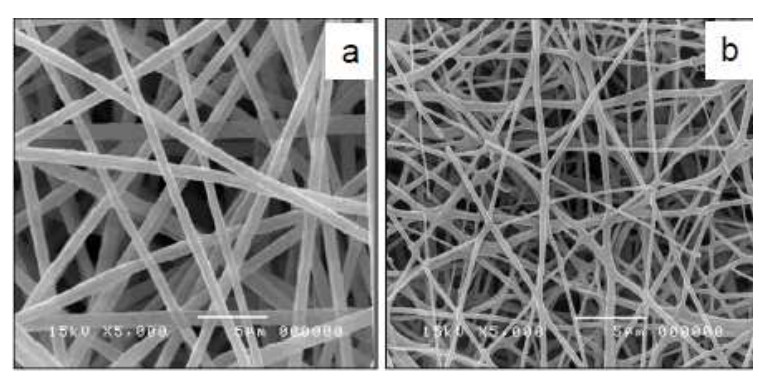

Fig. 1 Scanning electron microscope images of nonwoven nanofibre mats generated from electrospinning (a) polyvinylidene fluoride and (b) polylactic acid polymer solutions. Magnification $=5000 \times$. 


\section{Pollen germination tests}

The basal liquid medium for in vitro pollen germination consisting of $0.1 \mathrm{mM} \mathrm{H}_{2} \mathrm{BO}_{3}$ and $1 \mathrm{mM}$ $\mathrm{CaCl}_{2} \cdot 2 \mathrm{H}_{2} \mathrm{O}$ was modified after that of Mercado et $\mathrm{al}^{13}$. Unless specified otherwise, this basal medium was also supplemented with $20 \%(\mathrm{w} / \mathrm{v})$ sucrose and then the $\mathrm{pH}$ of the medium was adjusted to 5.7 before sterilization in an autoclave at $121^{\circ} \mathrm{C}$ and 15 psi for $20 \mathrm{~min}$. After this, $10 \mathrm{ml}$ of the liquid medium was poured into a sterile tissue culture dish $(60 \times 15 \mathrm{~mm}$, Becton Dickinson Labware, NJ, USA). If required, agar $(0.8 \%, w / v)$ was added to the basal medium before the sterilization step. Other pollen germination substrates investigated were the different electrospun nanofibre mats generated as described earlier here. A nanofibre mat was floated on the surface of the autoclaved basal medium by laying an edge of it down onto the surface of the medium first and then tenderly sliding the nanofibre mat down onto the surface of the medium.

Pollen grains were brushed off from the anthers of yellowish orange flowers and then dusted onto the surface of a germination support matrix (liquid, agar or a nanofibre mat). The dishes with pollen grains were moved carefully to a growth room for $24 \mathrm{~h}$ incubation at $30 \pm 1{ }^{\circ} \mathrm{C}$ in the dark. For each of the six replications of a germination support matrix, ten fields under a light microscope were observed. Pollen grains were considered to have germinated when the emerged pollen tubes were longer than the diameter of the pollen ${ }^{14}$. Pollen tube length measurements were made after $24 \mathrm{~h}$ of germination with the aid of an ocular micrometre as viewed under a light microscope.

\section{Statistical analysis}

ANOVA of the data on germination percentages and pollen tube length was carried out. Based on the requirement, Duncan's MRT test was carried out at the significance level of $p<0.05$.

\section{RESULTS}

Generally, the yellow petals of the A. hexapetalus flowers develop from the green bud stage in about 15 days and then they turn yellowish orange one day later as a normal course of floral development under natural growth conditions in Thailand. Under a light microscope, pollen grains from yellow and yellowish orange florets were of similar sizes after harvest and there was no change upon rehydration (unpublished observations).

The percentages of pollen germination in vitro
Table 2 In vitro germination tests using A. hexapetalus pollen from yellow and yellowish orange flowers on agargelled media supplemented with different concentrations of sucrose.

\begin{tabular}{lcc}
\hline Sucrose (\%) & \multicolumn{2}{c}{ Germination (\%)* } \\
\cline { 2 - 3 } & Yellow flower & Yellowish orange flower \\
\hline 5 & $20.42 \pm 0.63$ & $27.96 \pm 0.46$ \\
10 & $24.06 \pm 0.46$ & $34.80 \pm 0.45$ \\
20 & $26.12 \pm 0.41$ & $50.35 \pm 0.47$ \\
\hline * Data are mean \pm standard error of 60 replications and \\
\multicolumn{2}{l}{ all the values are significantly different (ANOVA, $p<$} \\
0.05).
\end{tabular}

Table 3 In vitro germination tests using pollen from yellowish orange flowers in liquid medium or on different electrospun nanofibre mats floating on the surface of the liquid medium supplemented with $20 \%$ sucrose.

\begin{tabular}{lcc}
\hline Support & $\begin{array}{c}\text { Thickness of } \\
\text { nanomats }(\mu \mathrm{m})\end{array}$ & $\begin{array}{c}\text { Germination } \\
(\%)^{*}\end{array}$ \\
\hline Liquid medium & - & $59.4 \pm 1.3^{\mathrm{d}}$ \\
PVDF1 & $8 \pm 2$ & $66.1 \pm 1.2^{\mathrm{ef}}$ \\
PVDF4 & $8 \pm 2$ & $70.22 \pm 0.92^{\mathrm{g}}$ \\
PLA1 & $8 \pm 2$ & $65.6 \pm 1.3^{\mathrm{e}}$ \\
PLA4 & $8 \pm 2$ & $68.5 \pm 1.4^{\mathrm{fg}}$ \\
PVDF1 & $18 \pm 2$ & $1.92 \pm 0.53^{\mathrm{a}}$ \\
PVDF4 & $18 \pm 2$ & $2.29 \pm 0.45^{\mathrm{a}}$ \\
PLA1 & $18 \pm 2$ & $0.30 \pm 0.21^{\mathrm{a}}$ \\
PLA4 & $18 \pm 2$ & $2.18 \pm 0.52^{\mathrm{a}}$ \\
\hline
\end{tabular}

* Data are mean \pm standard error of 60 replications and those sharing the same letter are not significantly different (ANOVA, $p<0.05$ ).

from the yellow $A$. hexapetalus flowers only improved slightly (from 20-26\%) with the increased concentrations of sucrose in the medium ranging from 5-20\% (Table 2). In contrast, there was about two-fold increase in the percentage of pollen $(50 \%)$ from yellowish orange flowers that germinated in the presence of $20 \%$ sucrose concentration as compared to that of $5 \%$ sucrose (Table 2). Based on these results, only pollen from the yellowish orange florets was used for further investigations.

Interestingly, the percentage of pollen (59\%) from yellowish orange $A$. hexapetalus flowers germinated in liquid medium supplemented with $20 \%$ sucrose was significantly higher than those pollen grains that were germinated in agar-gelled medium with $50 \%$ sucrose of otherwise the same composition (Table 2 and Table 3; Fig. 2a and Fig. 2b). Another experiment was also carried out to investigate 


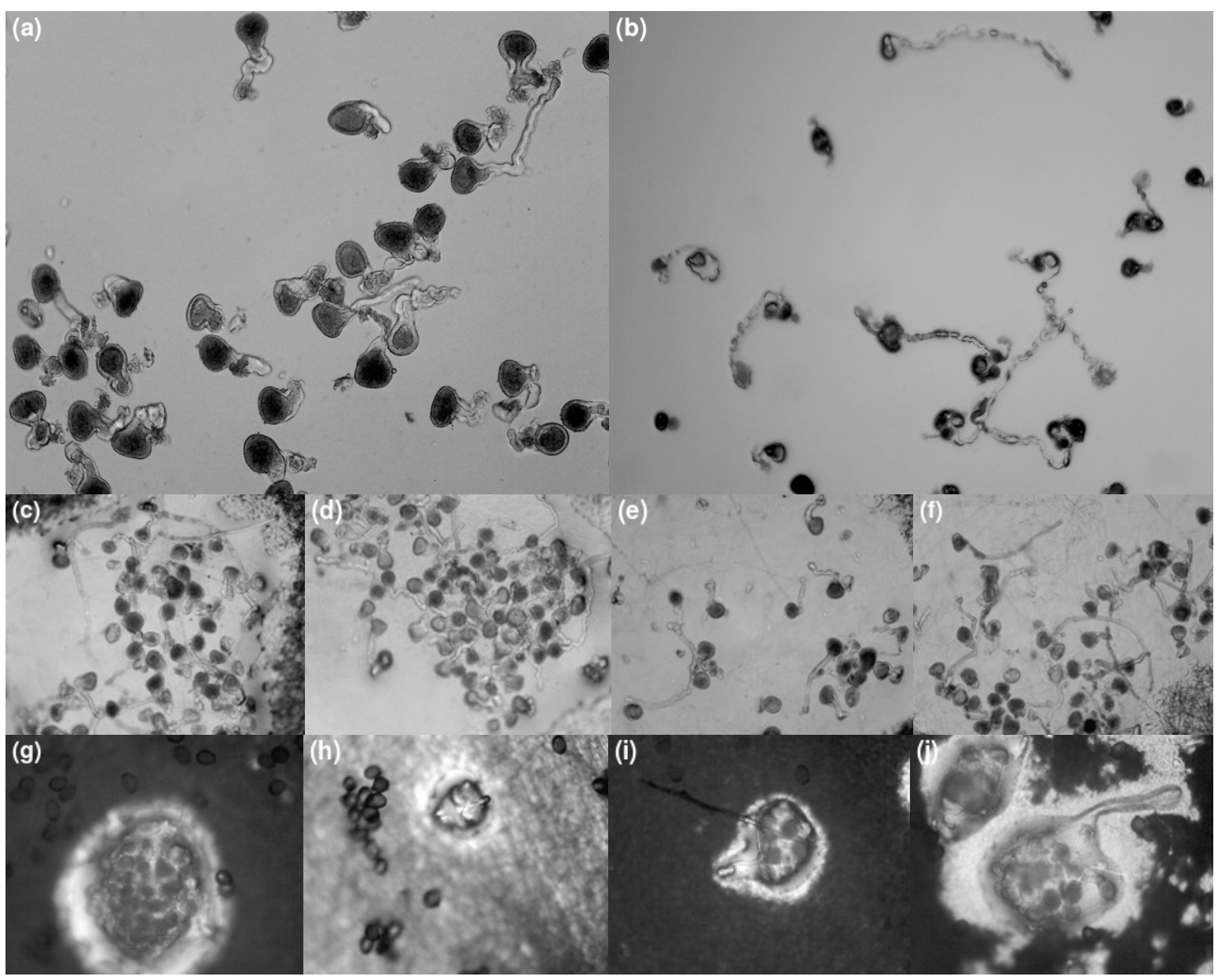

Fig. 2 Germination of Artabotrys hexapetalus pollen (a) in the basal liquid medium supplemented with $20 \%$ sucrose, (b) on the surface of agar-gelled basal liquid medium supplemented with $20 \%$ sucrose, and (c-j) on the surfaces of different electrospun nanofibre mats of either $8 \pm 2 \mu \mathrm{m}$ or $18 \pm 2 \mu \mathrm{m}$ floating on the basal liquid medium supplemented with 20\% sucrose: (c) PVDF1, $8 \pm 2 \mu \mathrm{m}$; (d) PVDF4, $8 \pm 2 \mu \mathrm{m}$; (e) PLA1, $8 \pm 2 \mu \mathrm{m}$; (f) PLA4, $8 \pm 2 \mu \mathrm{m}$; (g) PVDF1, $18 \pm 2 \mu \mathrm{m}$; (h) PVDF4, $18 \pm 2 \mu \mathrm{m}$; (i) PLA1, $18 \pm 2 \mu \mathrm{m}$; (j) PLA4, $18 \pm 2 \mu \mathrm{m}$.

the germination of $A$. hexapetalus pollen on different types, patterns, and thicknesses of electrospun nanofibre mats floated on the liquid medium were initiated (Table 3; Fig. 2c-j). The best physical support for in vitro germination of $A$. hexapetalus pollen was the $8 \mu \mathrm{m}$ thick PVDF4 or PLA4 nanofibre mats, resulting in about $70 \%$ germination (Table 3 ). The use of the nonwoven PVDF1 and PLA1 nanofibre mats, both $8 \mu \mathrm{m}$ thick, resulted in slightly lower pollen germination (about 66\%) than the patterned ones of the same thickness. Thicker $(18 \mu \mathrm{m})$ electrospun nonwoven and patterned PVDF and PLA nanofibre mats were found to inhibit germination of $A$. hexapetalus pollen (Table 3 ).

Pollen tube length measurements revealed that pollen tube growth (length) on the surface of agar- gelled medium $(392 \mu \mathrm{m})$ was more than double compared to those that was grown in liquid medium $(142 \mu \mathrm{m})$ (Table 4). This was contrary to the pollen germination results comparing liquid and agar medium (Table 2 and Table 3). Furthermore, it was found that PLA4 and PVDF4, both of $8 \mu \mathrm{m}$ thick, were not only better for pollen germination but were also more beneficial for pollen tube growth (pollen tube length being about 30 and $40 \%$, respectively, longer than those on agar-gelled medium).

\section{DISCUSSION}

In vitro germination of pollen has been extensively used for studying the genetical, physiological, biochemical and cytochemical characters for a wide range of plant species belonging to different fami- 
Table 4 In vitro germination tests using pollen tube length after $24 \mathrm{~h}$ of germination on different germination supports.

\begin{tabular}{lc}
\hline Support & Pollen tube length $(\mu \mathrm{m})$ \\
\hline Agar medium & $392 \pm 16$ \\
Liquid medium & $142 \pm 15$ \\
PLA4 (8 $\pm 2 \mu \mathrm{m}$ thickness) & $502 \pm 15$ \\
PVDF4 $(8 \pm 2 \mu \mathrm{m}$ thickness $)$ & $545 \pm 13$ \\
\hline
\end{tabular}

Data are mean \pm standard error of 30 replications and all the values are significantly different (ANOVA, $p<$ 0.05).

lies. Pollen grains play a vital role in crop improvement programmes and have relative importance not only on fruit set but also on the flower-flower and flower-pollen interaction. The prime objective of the study was to investigate the possible innovative utility of electrospun nanofibre mats as a suitable physical support matrix for pollen germination in vitro of $A$. hexapetalus. The pollen germination medium was modified after that of Mercado et $\mathrm{al}^{13}$ and used without further optimization of the main chemical ingredients except sucrose concentrations.

In the present investigation, the type of A. hexapetalus pollen to be used was determined since pollen grains were produced by florets in two closely related flower developmental stages: yellow and yellowish orange florets. It is widely accepted that pollen from different plant species may require a specific amount of sucrose in an artificial medium for germination in vitro. This exogenous sucrose requirement has been found to play an important role as a nutritive resource and osmoregulator for pollen germination ${ }^{12,15,16}$. The germination percentage of pollen from yellowish orange increased with the increasing concentrations of sucrose in the medium, suggesting that endogenous physiological differences of pollen might also play a role in regulation of pollen germination.

It has also been observed that the agar-based medium did not provide a sufficient physical support matrix for germination of $A$. hexapetalus on different types, patterns, and thickness of electrospun nanofibre mats floated on the liquid medium (Table 3; Fig. $2 \mathrm{c}-\mathrm{j}$ ). The results revealed that the appropriate electrospun nanofibres such as $8 \mu \mathrm{m}$ thick PVDF4 was found to be a promising novel alternative germination substrates that could substantially improve A. hexapetalus pollen germination in liquid or agarbased medium. Under natural conditions, pollen grains germinate on the stigmatic surface which is a biologically complex solid support matrix ${ }^{17}$. Liquid medium supports pollen hydration but it might not be the same as the fluid microenvironment on the surface of complex solid matrix. It is possible that certain nanofibre mats could better resemble the complex nature of that of the biologically complex stigmatic surface. The precise basis for the beneficial effects of the $8 \mu \mathrm{m}$ thick PVDF4 for pollen germination and tube growth of $A$. hexapetalus over other types of physical supports, however, remains to be established.

Conclusively, electrospun nanofibre mats, particularly $8 \mu \mathrm{m}$ thick PVDF4, influenced the in vitro pollen germination and tube growth of A. hexapetalus. Pollen quality is also better at yellowish orange coloured stage of petals in this plant. Further research needs to be carried out to optimize the medium composition and to see if these novel scaffolds could also be used to obtain better pollen germination and pollen tube growth in other plants besides $A$. hexapetalus.

Acknowledgements: We would like to thank Assoc. Prof. Boonyuen Kijwijan for his kind provision of sterile tissue culture dishes used in this work.

\section{REFERENCES}

1. Selak GV, Perica S, Ban SG, Poljak M (2013) The effect of temperature and genotype on pollen performance in olive (Olea europaea L.). Sci Hort 156, $38-46$.

2. Steinhorst L, Kudla J (2013) Calcium - a central regulator of pollen germination and tube growth. Biochim Biophys Acta Mol Cell Res 1833, 1573-81.

3. Taylor LP, Hepler PK (1997) Pollen germination and tube growth. Annu Rev Plant Physiol Plant Mol Biol 48, 461-91.

4. Vasil IK (1987) Physiology and culture of pollen. In: Bourne GH, Jeon KW, Friedlander M (eds) Pollen: Cytology and Development, International Review of Cytology vol. 107, pp 127-74.

5. Rodriguez-Enriquez MJ, Mehdi S, Dickinson HG, Grant-Downton RT (2013) A novel method for efficient in vitro germination and tube growth of Arabidopsis thaliana pollen. New Phytol 197, 668-79.

6. Leins P, Erbar C (1996) Early floral developmental studies in Annonaceae. In: W. Morawetz H, Winkler (eds) Reproductive Morphology in Annonaceae, Österreich Akademie der Wissenschaften, Vienna, Austria, pp 1-27.

7. Svoma E (1997) Seed development and function in Artabotrys hexapetalus, (Annonaceae). Plant Systemat Evol 207, 205-23.

8. Tsou CH, Johnson DM (2003) Comparative develop- 
ment of aseptate and septate anthers of Annonaceae. Am J Bot 90, 832-48.

9. Xu F, de Craene LR (2010) Floral ontogeny of Annonaceae: evidence for high variability in floral form. Ann Bot 106, 591-605.

10. Yang F, Murugan R, Wang S, Ramakrishna S (2005) Electrospinning of nano/micro scale poly(L-lactic acid) aligned fibers and their potential in neural tissue engineering. Biomaterials 26, 2603-10.

11. Chanunpanich N, Lee B, Byun H (2008) A study of electrospun PVDF on PET sheet. Macromol Res 16, 212-7.

12. Bodhipadma K, Noichinda S, Yadbuntung I, Buaeiam W, Leung DWM (2010) Comparison of in vitro and in vivo inflorescence of common cockscomb (Celosia argentea var. cristata). Sci Asia 36, 68-71.

13. Mercado JA, Fernández-Muñoz R, Quesada MA (1994) In vitro germination of pepper pollen in liquid medium. Sci Hort 57, 273-81.

14. Kakani VG, Prasad PVV, Craufurd PQ, Wheeler TR (2002) Response of in vitro pollen germination and pollen tube growth of groundnut (Arachis hypogaea L.) genotypes to temperature. Plant Cell Environ 25, 1651-61.

15. Ali T, Ali SI (1996) Effect of sugar concentration on pollinium germination in some members of Asclepiadaceae. Pakistan J Bot 28, 161-5.

16. Baloch MJ, Lakho AR, Bhutto H, Solangi MY (2001) Impact of sucrose concentrations on in vitro pollen germination of okra, Hibiscus esculentus. Pakistan $J$ Biol Sci 4, 402-3.

17. Firon N, Nepi M, Pacini E (2012) Water status and associated processes mark critical stages in pollen development and functioning. Ann Bot 109, 1201-13. 